\title{
La beauté ajoutée cyclique
}

\section{Christophe Pradeau}

\section{(2) OpenEdition}

\section{Journals}

Édition électronique

URL : https://journals.openedition.org/genesis/1621

DOI : 10.4000/genesis. 1621

ISSN : 2268-1590

\section{Éditeur :}

Presses universitaires de Paris Sorbonne (PUPS), Société internationale de génétique artistique littéraire et scientifique (SIGALES)

\section{Édition imprimée}

Date de publication : 9 mai 2016

Pagination : $37-51$

ISBN : 9791023105315

ISSN : 1167-5101

\section{Référence électronique}

Christophe Pradeau, «La beauté ajoutée cyclique », Genesis [En ligne], 42 | 2016, mis en ligne le 05 juin 2017, consulté le 17 janvier 2023. URL : http://journals.openedition.org/genesis/1621 ; DOl : https:// doi.org/10.4000/genesis.1621 


\title{
La beauté ajoutée cyclique
}

\author{
Christophe Pradeau
}

$\mathrm{D}$ ans une page célèbre de La Prisonnière, Proust invite à voir dans l'unité multiple cyclique, façon souple et mobile d'embrasser l'ambition monumentale, la forme même du XIXe siècle, celle dans laquelle l'époque s'est reconnue, lieu réflexif qu'elle oppose à l'énigme du devenir, depuis lequel elle questionne le mystère des identités traversées par le temps, les enjeux de la non-coïncidence de soi à soi et les équivoques des héritages. Et de fait, le cycle, tout au long du siècle, a prêté sa forme instable mais aussi sa dramaturgie - cloisons qui tombent ou se déplacent, déplacements de perspectives, changements à vue qui dépaysent le familier et réinventent le monde - aux existences individuelles et aux romans familiaux, au Grand Récit national mais aussi aux épopées de la longue durée, aux spéculations poétiques ou philosophiques sur la solidarité transhistorique de l'espèce. Aussi peut-on y voir la forme même de l'intelligibilité historique telle qu'elle s'est imposée aux contemporains de Balzac et de Zola, de Hugo et de Wagner, de Cuvier et de Darwin, de Ballanche, de Michelet ou d'Hegel.

Si l'idée de cycle informe l'identité narrative du siècle, elle n'en est pas moins associée à Balzac comme à une figure fondatrice, et le roman est largement considéré comme le lieu de son invention. On sait que Balzac assigne au genre romanesque l'ambition d'écrire l'histoire $\mathrm{du}$ «présent qui marche ${ }^{1}$ », ce qui est une façon de lui découvrir une vocation, autrement dit une dignité cognitive, de justifier sa prétention à prendre place aux côtés de l'histoire et de la philosophie, ce qui implique aussi, comme le soulignent les préfaces d'accompagnement de La Comédie humaine, de surmonter les difficultés techniques que pose l'imitation d' «un modèle extrêmement remuant et difficile à faire tenir en place ${ }^{2} \gg$. La technique du retour des personnages participe d'une ambition que l'on pourrait qualifier de cinématographique; elle se justifie, en tout premier lieu, en effet, par la nécessité où se trouve le romancier, s'il veut réaliser le mouvement, d'inventer un mode de composition qui permette de représenter l'ondoiement du devenir, ce qui peut impliquer, dans le cas de Balzac, de renoncer à l'ordre chronologique, d'accepter que ses romans se constituent, reliés entre eux, solidarisés par le jeu dynamique de carrefours, en « un jardin aux sentiers qui bifurquent ${ }^{3}$ », qu' ils se confondent, du moins en apparence, avec une figure de l'égarement comme le labyrinthe, alors même que leur ambition est de donner forme et lisibilité au monde.

Le propos de Proust est informé en profondeur par l'ambition balzacienne, reconnue comme fondatrice, mais il excède le genre romanesque, en localisant dans le mot cycle l'énigme d'une analogie de structure, la congruence d'une époque et d'une forme. Si le roman,

1.H. de Balzac, préface d'Une fille d'Ève [1839], La Comédie humaine [désormais CH], dir. P.-G. Castex, Paris, Gallimard, coll. «Bibliothèque de la Pléiade», t. II, p. 263.

2.Ibid., p. 265.

3. La nouvelle de Borges est érigée en métaphore totalisante par J. Gleize, dans un article sur le mode de composition de l'œuvre balzacienne : «La Comédie humaine : un livre aux sentiers qui bifurquent», Poétique, $\mathrm{n}^{\circ} 115$, septembre 1998, p. 259-271. 


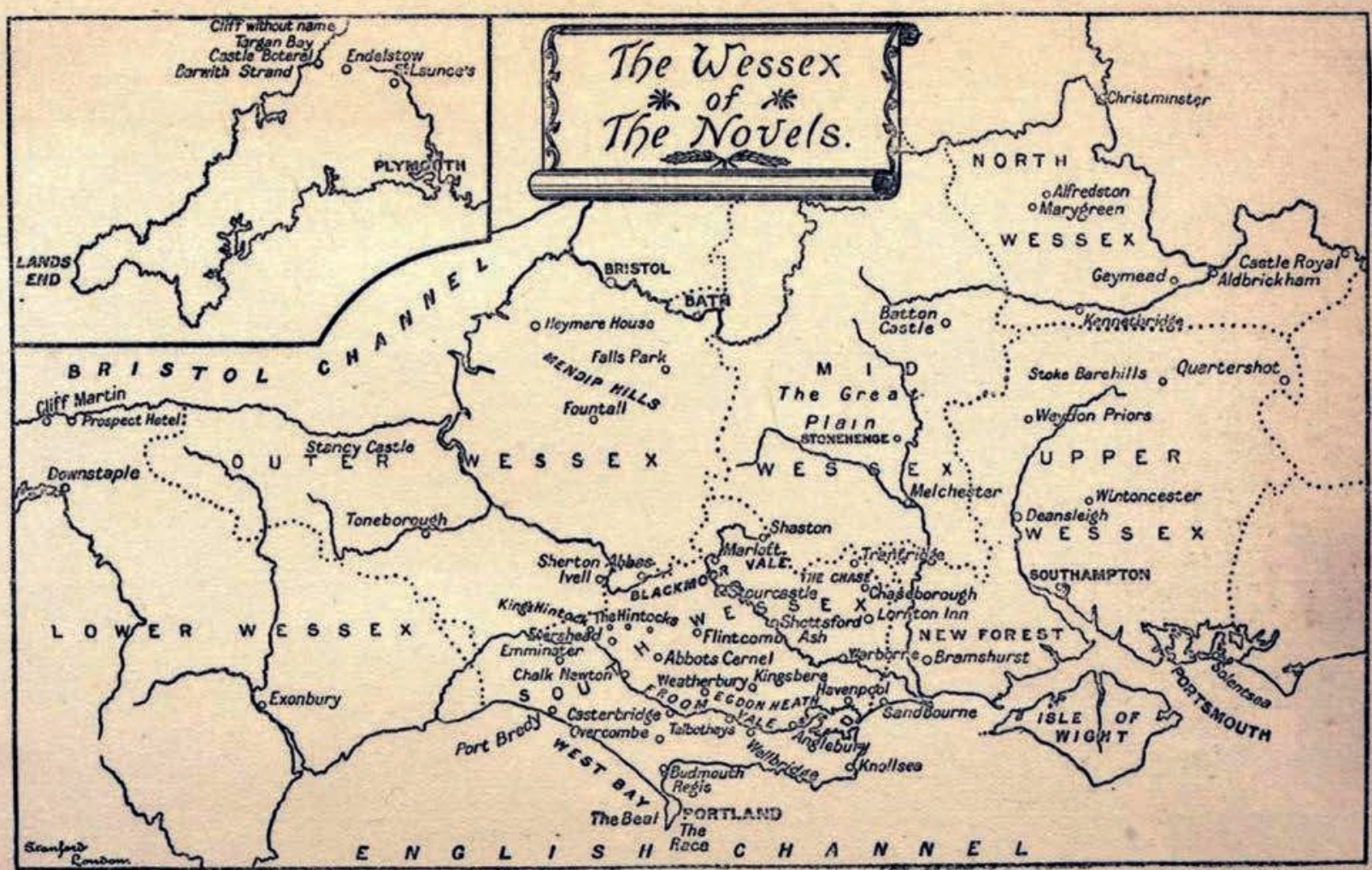

Fig. 1 : Carte des Wessex Novels de Thomas Hardy genre roturier, sans histoire ni qualités, est l'idiome par excellence de l'individu démocratique, le truchement du temps laïcisé du grand désert d'hommes, Proust pose qu'il partage avec toutes les entreprises de figuration mémorable du XIX ${ }^{\mathrm{e}}$ siècle, l'ambition d'inventer une unité qui serait à la démesure des villes modernes, ce qui impose de rompre avec l'impératif aristotélicien de l'appréhension synoptique, ce confort perceptif et mémoriel dont La Poétique fait la condition de la relation esthétique. Une œuvre cyclique ne peut, comme le voudraient les préceptes aristotéliciens, être embrassée du regard : elle est toujours, aussi quadrillée, aussi cadastrée, aussi lisible soit-elle, un labyrinthe. Elle demande à être réalisée en soi, avec l'œil de l'esprit, entretenue par une fréquentation mémorieuse, étayée, Balzac lui-même en suggère l'idée, par des aide-mémoire, répertoires biographiques des personnages, comme en composent les «balzaciens » de la Belle Époque, comme ceux, établis par Lise Jules-Romains, qui accompagnent la parution échelonnée des Hommes de bonne volonté, mais aussi cartes comme chez Thomas Hardy (fig. 1) ou Faulkner (fig. 2) - dont les romans et les nouvelles forment des cycles dont le principe unitaire n'est pas le retour des personnages mais la structure de monde d'un pays imaginaire -, arbres généalogiques comme chez Zola (fig. 3), tableaux synoptiques à usage public ou privé comme chez Roger Martin du Gard.

Le présent article s'organise en deux parties. La première essaie de dégager quelques-uns des enjeux de l'invention, à la fin du XIX'e siècle, de l'idée de cycle, en dépliant brièvement l'héritage du mot autour duquel cristallise l'ambition de représenter le monde dans une œuvre réticulaire à prétention totalisante. Suivent, dans une seconde partie, deux fables «génétiques » où s'exposent, depuis l'enveloppement d'un chantier perçu par l'écrivain comme coextensif à son existence, quelque chose du propre des entreprises cycliques, une certaine façon de gérer le faire, d'inventer de l'intérieur, depuis l'intimité d'un englobant sans ailleurs, l'espace au large d'une prolifération susceptible de faire monde. 


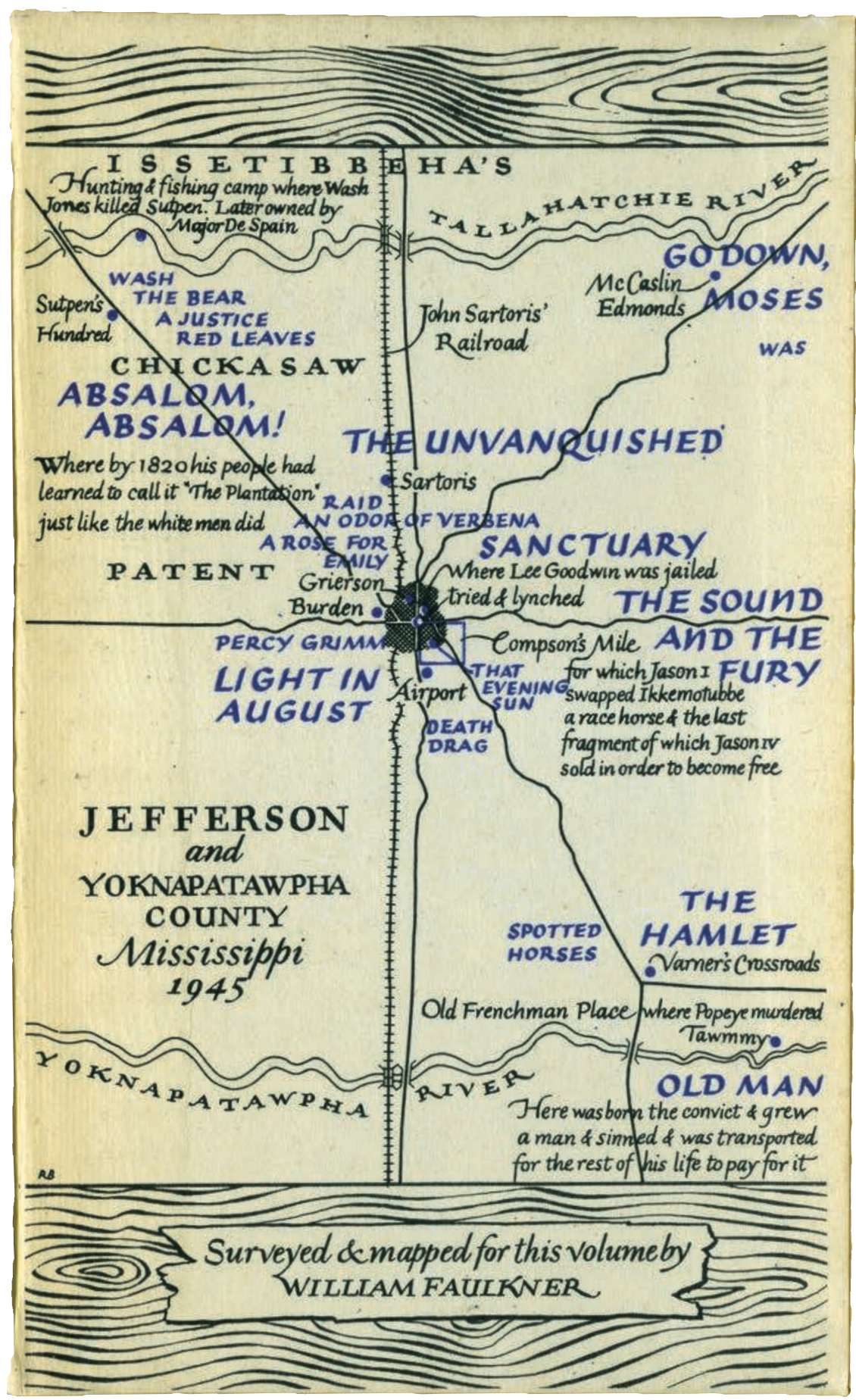

Fig. 2 :

Carte du Yoknapatawpha County, dessinée par William Faulkner en 1946 pour The Portable Faulkner (éd. Malcolm Cowley, Viking Press) 


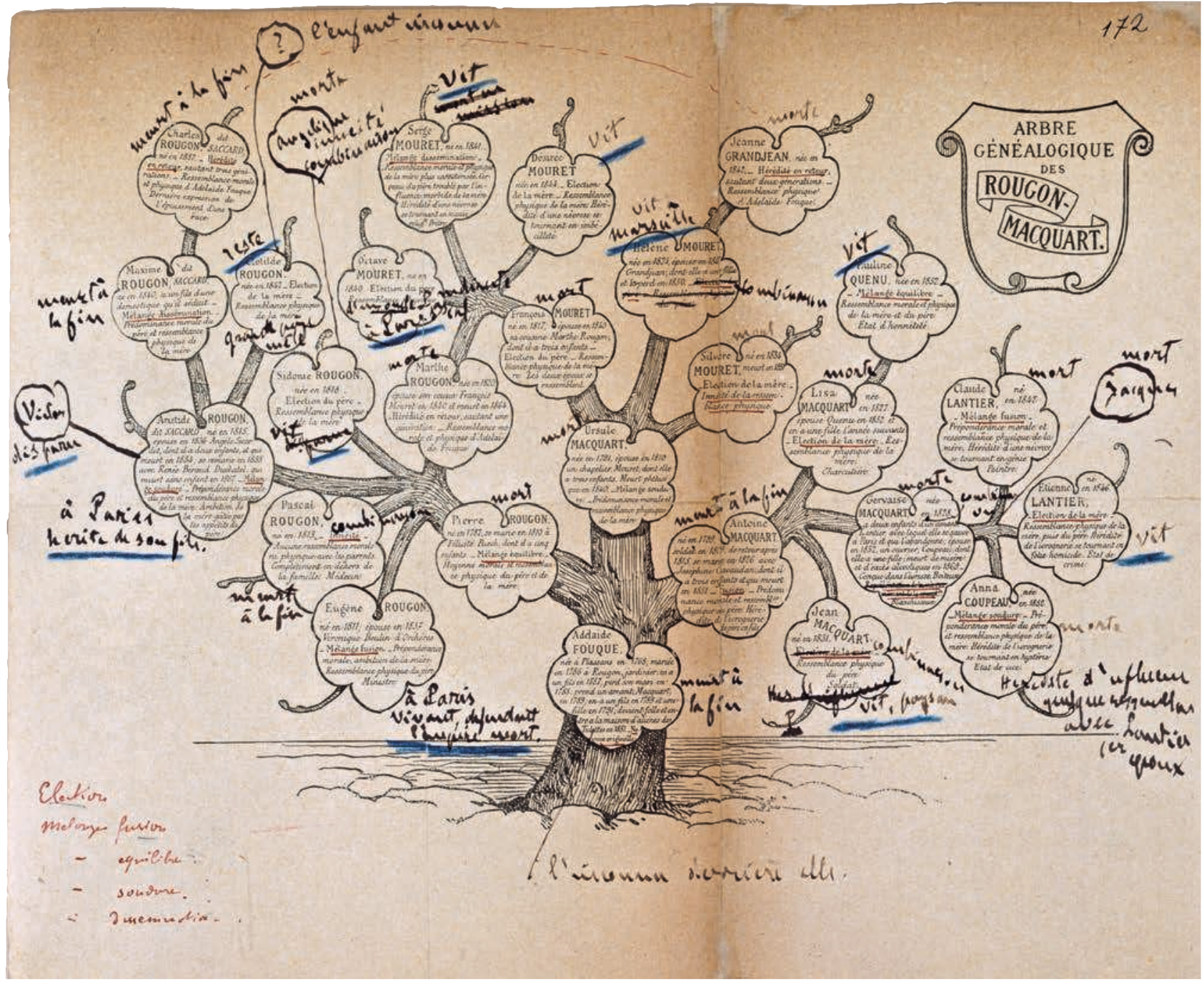

Fig. 3 : Émile Zola, Arbre généalogique des Rougon-Macquart, état de 1878, avec des corrections autographes réalisées en 1892 (BnF, NAF 10290, fo 172)

L'héritage d'un mot : cycle
Le texte de La Prisonnière retient quatre figures, le Balzac de La Comédie humaine, le Hugo de La Légende des siècles, le Michelet de l'Histoire de France et le Wagner du Ring, qui ont en commun - si du moins l'on accepte le scénario génétique proposé par le narrateur, qui résulte d'une information factuelle partiellement erronée 4 - d'avoir découvert le principe unitaire de leur œuvre dans l' «après coup» d'une «autocontemplation», expérience existentielle autant qu'esthétique que l'auteur de la Recherche nomme «illumination rétrospective» :

Wagner [...] s'apercevant tout à coup qu'il venait de faire une Tétralogie, dut éprouver un peu de la même ivresse que Balzac quand celui-ci, jetant sur ses ouvrages le regard à la fois d'un étranger et d'un père, trouvant à celui-ci la pureté de Raphaël, à cet autre la simplicité de l'Évangile, s'avisa brusquement en projetant sur eux une illumination rétrospective qu'ils seraient plus beaux réunis en un cycle où les mêmes personnages reviendraient et ajouta à son œuvre, en ce raccord, un coup de pinceau, le dernier et le plus sublime ${ }^{5}$.

4. Voir notamment J.-J. Nattiez, Proust musicien, Paris, Christian Bourgois, 1999, p. 45 sq.

5. M. Proust, La Prisonnière, dans À la recherche du temps perdu [désormais RTP], dir. J.-Y. Tadié, Paris, Gallimard, coll. «Bibliothèque de la Pléiade», 1988, t. III, p. 666. 
C'est en se retournant sur leurs œuvres, en s'en faisant les lecteurs ou les spectateurs, que Balzac, Hugo, Michelet et Wagner sont touchés par le sentiment que leurs ouvrages trouveraient un surcroît de beauté à être appréhendés comme les parties constitutives d'un cycle. Comme, dans la Recherche, Elstir est la peinture en personne, Balzac est considéré à la fois en lui-même et comme chef de file du roman, de même que Hugo incarne la poésie, Michelet l'histoire et Wagner la musique. Le quadrige représente, en effet, le pouvoir de figuration mémorable d'un siècle qu'il entraîne derrière lui, qu'il réalise et nous transmet sous la forme d'un passé actualisable, autrement dit participable, un passé qui est aussi, en puissance, une manière de présent. C'est là l'une des définitions possibles de l'héritage et c'est, de fait, celui que Proust se reconnaît et dans lequel il identifie la donne des hommes et des femmes de sa génération. En conjoignant ces quatre grandes figures, Proust cherche à décrire l'ombre portée du XIX $\mathrm{X}^{\mathrm{e}}$ siècle, ou, si l'on transpose la métaphore optique en termes psychologiques, la nature double du sentiment d'admiration 6 , qui fortifie et accable, portant en lui émulation et abattement. La grande méditation esthétique de La Prisonnière est à la fois une réflexion poétique sur le cycle et une appréciation de l'héritage du XIX ${ }^{\mathrm{e}}$ siècle.

Le mot cycle porte en lui tout le poids d'un héritage, et ce à plus d'un titre. C'est le nom que Proust donne au siècle précédent, à l'héritage glorieux du siècle de Balzac et de Wagner; mais c'est aussi un mot qui révèle la prétention du roman, genre amnésique, sans règle ni tradition, dont on n'écrit ni n'enseigne l'histoire, à revendiquer, c'est l'un des enjeux de l'idée de Comédie humaine, l'héritage de la grande poésie épique et didactique. Le mot cycle n'est pas, dans son acception esthétique, employé par Balzac, pas plus qu'il ne l'est, quatre décennies plus tard, par le Zola des «dossiers préparatoires », celui qui se projette dans le chantier-monde des Rougon-Macquart. De fait, il faut attendre la fin du siècle pour que le mot s'impose progressivement dans son acception esthétique d' «œuvre composée» ou «à manifestation partielle ${ }^{7}$ ». Le mot cycle connaît, au tournant des $\mathrm{XIX}^{\mathrm{e}}$ et $\mathrm{XX}^{\mathrm{e}}$ siècles, une fortune inattendue, qu'il doit à son adoption par les médiévistes, à un moment où, dans le contexte géopolitique de la Revanche, la littérature du Moyen Âge devient un enjeu politique. Les philologues qui contribuent à reconfigurer l'identité nationale en mettant au jour la geste de Charlemagne ou le roman de Lancelot, sont frappés de reconnaître dans les enfances de la littérature française un principe de liaison analogue à celui que nomme, dans le monde grec, le mot kuklos. En préférant employer, plutôt que des termes endogènes, celui de cycle, pour désigner les vastes ensembles épiques ou romanesques, les médiévistes ont l'ambition de désenclaver la littérature médiévale, plaidant de facto pour l'invention d'un scénario historique qui tiendrait ensemble Homère et Turold, Virgile et Chrétien de Troyes. Le mot cycle qui désigne, à l'époque hellénistique, un corpus canonique de seize épopées, incluant l'Iliade et l'Odyssée, est bien connu des lycéens du Second Empire et de la Troisième République et, plus généralement, de ceux que Jean Paulhan appelle le moyen public cultivé. Le cas du Cycle épique est exemplaire de ce que Judith Schlanger a appelé «la présence des œuvres perdues $^{8}$ ». Seules nous ont été transmises les deux épopées attribuées à Homère, et quelques

6. Voir J. Schlanger, La Mémoire des æuvres, Lagrasse, Verdier, coll. «Poche», 2008 [1992], p. 88 sq.

7. G. Genette, L'Euvre de l'art, Paris, Éditions du Seuil, coll. « Poétique », 1994, t. I, «Immanence et transcendance », p. 239 sq.

8. J. Schlanger, Présence des ceuvres perdues, Paris, Hermann, coll. «Savoir lettres », 2010. 
fragments de leurs continuations, mais le Cycle épique, dont on trouve des résumés dans les manuels et les dictionnaires encyclopédiques du long XIX ${ }^{\mathrm{e}}$ siècle, informe obscurément notre lecture d'Homère.

C'est la génération de Proust qui adopte résolument le terme, l'empruntant aux philologues et aux historiens de l'Antiquité, pour l'appliquer à la littérature qui s'écrit au présent. Le mot cycle n'est pas un mot de poète : il appartient au vocabulaire de l'érudition, à l'histoire de la transmission et à celle des supports textuels ; c'est un mot de scoliaste ou de codicologue. Il appartient aussi, il est vrai, à la tradition des arts poétiques mais cantonné dans les marges, où il joue le rôle ingrat du contre-modèle : quand les poéticiens des siècles classiques rencontrent chez Horace le continuateur d'Homère, le scriptor cyclicus, ils comprennent et traduisent le mauvais poète 9 . Appliqué aux chansons de geste ou aux grands romans du XIII siècle, le mot cycle désigne moins l'œuvre elle-même que son support, le manuscrit dit cyclique, ainsi qualifié parce qu'il rassemble la matière d'un univers fictionnel, en compilant des textes d'auteurs différents. Le mot qualifie davantage, somme toute, un geste d'éditeur ou de lecteur que de poète, ou alors, parce que le terme désigne quand même aussi un mode de composition, celui d'un poète-lecteur, la part de lecture, lecture des autres et de soi-même, qu'il y a dans l'acte d'écrire. Il est significatif, à cet égard, que le meilleur ouvrage de synthèse consacré à la «cyclification» de la littérature romanesque médiévale porte comme titre Lectures cycliques ${ }^{10}$. Appliqué à des ensembles non plus éditoriaux mais auctoriaux, à ces œuvres que Northrop Frye propose d'appeler des «One-Man-Bibles ${ }^{11}$ », en l'espèce les œuvres-mondes de la grande génération romantique, le mot change de valeur et de portée : il a désormais partie liée avec le mystère de la création et devient dès lors, à une époque qui participe encore largement de l'héroïsation romantique du geste créateur, un enjeu primordial, le lieu stratégique où il convient de se situer pour questionner la vocation du roman ou, plus radicalement encore, la réalité de l'art.

Le retour des personnages est une technique, mais c'est d'abord une idée, chez Balzac assurément, chez qui elle survient dans l'ivresse incrédule de la première fois, mais aussi chez les romanciers qui la lui emprunteront. Ou du moins, suggère Proust, le retour des personnages devrait conserver en lui, en devenant une technique, quelque chose de la grâce lumineuse de l'idée, mais, de même que la mystique finit en politique, l'idée de faire revenir ses personnages semble condamnée à se dégrader en technique. C'est tout l'enjeu de la réflexion proustienne sur «l'après coup », qui désigne le moment de l'invention comme la scène qui décide de la portée du geste cyclique, simple «habileté vulcanienne» de fabricateur ou authentique fiat

9. Voir, par exemple, l'abbé Le Bossu, Traité du poëme épique, Paris, Chez Michel le Petit, 1675, 2 vol., t. I, p. 298. 10. P. Moran, Lectures cycliques. Le réseau inter-romanesque dans les cycles du Graal du XIIIe siècle, Paris, Champion, coll. «Nouvelle Bibliothèque du Moyen Âge», 2014. Voir aussi l'ouvrage collectif Cyclification. The Development of narrative cycles in the Chansons de Geste and the Arthurian Romances, Amsterdam / Oxford / New York / Tokyo, Koninklijke Nederlandse Akademie van Wetenschappen Verhandelingen, Afd. Letterkunde, Nieuwe Reeks, 1994.

11. N. Frye, Le Grand Code. La Bible et la littérature, traduit de l'anglais par C. Malamoud, Paris, Éditions du Seuil, 1984. 
$\operatorname{lux}{ }^{12}$. Le texte hésite entre deux interprétations. Il fait tout d'abord une lecture disqualifiante de l'absence de préméditation :

[J]e songeais combien tout de même ces œuvres participent à ce caractère d'être - bien que merveilleusement - toujours incomplètes, qui est le caractère de toutes les grandes œuvres du XIX ${ }^{\mathrm{e}}$ siècle; du XIX ${ }^{\mathrm{e}}$ siècle dont les plus grands écrivains ont manqué leurs livres, mais, se regardant travailler comme s'ils étaient à la fois l'ouvrier et le juge, ont tiré de cette autocontemplation une beauté nouvelle, extérieure et supérieure à l'œuvre, lui imposant rétroactivement une unité, une grandeur qu'elle n'a pas ${ }^{13}$.

Puis, en une manière de palinodie, le narrateur y découvre tout le contraire d'un défaut, une marque d'authenticité :

Unité ultérieure, non factice. Sinon, elle fût tombée en poussière comme tant de systématisations d'écrivains médiocres qui à grand renfort de titres et de sous-titres se donnent l'apparence d'avoir poursuivi un seul et transcendant dessein. Non factice, peut-être même plus réelle d'être ultérieure, d'être née d'un moment d'enthousiasme où elle est découverte entre des morceaux qui n'ont plus qu'à se rejoindre, unité qui s'ignorait, donc vitale et non logique, qui n'a pas proscrit la variété, refroidi l'exécution ${ }^{14}$.

Ultime tour d'écrou, la méditation se referme sur la possibilité d'une réévaluation de la place et de la valeur de la technique : «Peut-être, comme les oiseaux qui montent le plus haut, qui volent le plus vite, ont une aile plus puissante, fallait-il de ces appareils vraiment matériels pour explorer l'infini $[\ldots]^{15}$.»

C'est cette hésitation que je voudrais questionner dans la seconde partie du présent article, et que voudrait nommer la formule lui donnant son titre, forgée en condensant les rêveries du héros de la Recherche qui, assis à son piano, découvre dans la beauté ajoutée cyclique le principe unitaire du XIXe siècle. Je voudrais, pour cela, présenter deux petites fables, qui esquissent, de Balzac à Zola, de Proust à Jules Romains, une histoire du cycle romanesque envisagé comme tension entre idée et technique, entre l'avant-coup et l'après-coup, autrement dit, considéré depuis l'hypothèse d'un récit de genèse.

L'anecdote est célèbre, rapportée par Laure Surville, la sœur de Balzac. Nous sommes en 1833. Le romancier est à son domicile, rue Cassini, lorsque l'idée lui vient de «relier tous ses personnages pour en former une société complète». Balzac «accourt» aussitôt chez sa sœur, faubourg Poissonnière, pour partager sa «découverte» :

- Saluez-moi, nous dit-il joyeusement, car je suis tout bonnement en train de devenir un génie!

Il nous déroule alors son plan qui l'effrayait bien un peu; quelque vaste que fût son cerveau, il fallait

12. Voir les deux articles que D. Jullien a consacrés à la méditation esthétique de La Prisonnière : «La préface comme autocontemplation », Poétique, n 84, 1990, p. 499-508 et " "Fiat lux" ", Revue des sciences humaines, $n^{\circ} 235,1994$, p. 125-151. Voir aussi, de la même, «La cathédrale romanesque », Bulletin de la société des amis de Marcel Proust et des amis de Combray, ${ }^{\circ}$ 40, 1990, p. 43-57.

13. M. Proust, La Prisonnière, dans RTP, t. III, p. 666. Le premier tirage (1988) comporte une coquille («marqué » au lieu de «manqué»).

14. Ibid., p. 667.

15. Ibid., p. 667-668

\section{Première fable génétique : l'illumination rétrospective}


du temps pour y emménager ce plan-là ! [...] Que ce sera beau si je réussis ! disait-il en se promenant par le salon; il ne pouvait tenir en place, la joie resplendissait sur tous ses traits.

- Comme je me laisserai tranquillement traiter de faiseur de nouvelles à présent, tout en taillant mes pierres! Je me réjouis à l'avance de l'étonnement des myopes quand ils verront le grand édifice qu'elles formeront 16 !

Le récit de Laure Surville y insiste, le visage de Balzac est «illuminé» par la «joie», traduction d'une expérience intime de dilatation 17 : éclosion de l'œuvre qui s'élargit soudain aux dimensions inattendues d'un monde, avec tout ce que cela implique d'inépuisable, de réserve indéfinie d'inventions, mais aussi de saisissement devant le vertige d'un grand large brusquement ouvert en soi. L'invention, fruit de la plénitude d'un instant, s'éprouve avant tout comme un changement d'échelle : la succession contingente des livres épars, livres trop brefs, dispersés, écrits à sauts et à gambades, simples nouvelles prises dans le flux oublieux du commerce de la librairie, se découvre une unité, un principe cohésif, une certitude sur laquelle Balzac pourra faire fond pour se soustraire aux sables mouvants du présent en donnant ses livres pour une œuvre et plus encore un monument, de ceux dans l'ombre desquels s'écrivent les livres à venir; la vie besogneuse, obscure, taraudée par le doute et le ressentiment, du tâcheron littéraire, se ramasse puissamment sur elle-même pour s'évader hors de ses contours, dans la certitude anticipée, et impatiente de l'accomplissement, que le temps épisodique du quotidien ne pourra faire autrement désormais que de coïncider avec le temps plein des vies mémorables, vécues sous le soleil de la vocation.

L'illumination ne commande pas, à proprement parler, de faire table rase : elle ne participe pas, comme on pourrait le penser, d'une dramaturgie de la conversion ni même, à dire vrai, de ces départs à nouveaux frais qu'emblématise l'expression de vita nova, dramaturgie existentielle de la page qui se tourne. Elle est tout à la fois et inséparablement rétrospective et prospective. Balzac poursuivra sur son erre; il continuera, somme toute, à faire ce qu'il a toujours fait mais conforté par la promesse, qu'implique la métaphore lapidaire, que le temps quotidien désormais fera œuvre : le mince roman, la simple nouvelle, sont beaucoup plus que cela, du moment qu'ils sont perçus comme des «pierres», autrement dit comme la partie d'un tout monumental et pérenne où ils trouveront leur juste place. L'idée illuminante se caractérise par sa vertu décloisonnante. Elle est moins une puissance de rupture qu'un opérateur de liaison. Elle modifie l'éclairage et les perspectives de la scène où se joue le drame de la création, en magnifiant ce qui était déjà là, en le redéployant sur une scène élargie, dans l'espace dynamique d'un projet, qui se révèle comme tel a posteriori, alors même que l'on y est déjà plongé, depuis des mois, des années, en toute ignorance, ou le sachant, mais d'un savoir obscur.

L'authenticité de l'anecdote passe pour douteuse ${ }^{18}$. On a pu s'étonner, en effet, de la date avancée par Laure Surville : on comprend mal pourquoi Balzac aurait patienté près d'un an avant de mettre en œuvre sa «découverte», puisqu'il faut attendre décembre 1834, et la

16. L. Surville, Balzac, sa vie et ses euvres d'après sa correspondance, Paris, Librairie Nouvelle-JaccottetBourdilliat, 1858, p. 95-96.

17. Voir J.-L. Chrétien, La Joie spacieuse. Essai sur la dilatation, Paris, Éditions de Minuit, coll. « Paradoxe », 2007. 18. Voir par exemple la préface de S. Vachon à son édition du Père Goriot, Paris, Le Livre de Poche, coll. «Classiques de Poche », 1995, p. 359. 
publication du Père Goriot dans La Revue de Paris, pour que l'idée du retour des personnages soit érigée en système. Par ailleurs, l'idée de relier ses livres entre eux est ancienne chez Balzac, qui l'expérimente dès le début des années 1820, comme en témoigne la préface d'Annette et le criminel (1824), dans laquelle Balzac, sous le nom d'Horace de Saint-Aubin, prévient le lecteur que «pour bien juger de l'ouvrage, il faut absolument connaître les antécédents de la vie du principal personnage de ce tableau, et il faut pour cela avoir lu Le Vicaire des Ardennes », publié deux ans plus tôt. Si l'hypothèse d'une invention du retour des personnages par tâtonnements successifs semble préférable à celle d'une brusque illumination, il n'en demeure pas moins que l'anecdote résonne. Elle donne à rêver. On s'y arrête. On la reprend, comme si l'on soupçonnait qu'en elle se disait quelque chose des enjeux et de l'effet esthétique de l'invention balzacienne. L'anecdote mobilise ce que Judith Schlanger a appelé «le schéma de l'illumination ${ }^{19}{ }^{»}$, qui dramatise le processus psychologique de l'invention, en lui donnant l'éclat d'une révélation. La prégnance de ce scénario dans la littérature sur l'invention au XIX ${ }^{\mathrm{e}}$ siècle semble témoigner d'une attention nouvelle à la part du travail inconscient dans l'activité intellectuelle : le surgissement soudain de l'idée, qui s'impose comme un don, dans la plénitude d'un état de perfection, traduirait, en effet, le brusque passage d'un état à l'autre, la traduction dans la conscience verbalisée d'une idée mûrie dans les profondeurs. Proust est du nombre de ceux qui ont pris au sérieux l'anecdote de Laure Surville, qui ont fait confiance à son pouvoir de résonance, découvrant dans «le schéma de l'illumination» quelque chose comme le chiffre de l'art du XIX ${ }^{\mathrm{e}}$ siècle. Dans une large mesure, les pages de La Prisonnière sont, en effet, un long commentaire in absentia du texte de Laure Surville20. Je n'ai pas encore donné de précisions sur la scénographie de la méditation. Le narrateur, en l'absence d'Albertine, s'est mis au piano pour jouer la sonate de Vinteuil. Le texte, pendant une dizaine de pages, mime le «cours de[s] rêveries » du narrateur ${ }^{21}$, ce «cheminement en étoile» dont parle Bachelard 22 , fait de soudaines bifurcations, de retours en arrière, qui est comme la flânerie de la pensée. Dans cet état de profonde disponibilité, une mesure de la sonate, maintes fois entendue pourtant, familière, frappe soudain le narrateur par la ressemblance qu'elle entretient avec un thème du Tristan de Wagner : «parfois, l'attention éclaire différemment des choses connues pourtant depuis longtemps et où nous remarquons ce que nous n'y avions jamais vu 23 ». Soucieux d'analyser l'événement perceptif que représente l'appel d'air soudain d'un dépaysement dans l'enveloppement du familier, le narrateur convoque une comparaison empruntée à la pensée de l'hérédité : «En jouant cette mesure, et bien que Vinteuil fût là en train d'exprimer un rêve qui fût resté tout à fait étranger à Wagner, je ne pus m'empêcher de murmurer : "Tristan!" avec le sourire qu'a l'ami d'une famille retrouvant quelque chose de

19. J. Schlanger, L'Invention intellectuelle, Paris, Fayard, 1983, p. 46-47.

20. Dans les fragments du Contre Sainte-Beuve que l'on peut considérer comme des avant-textes de la méditation esthétique de La Prisonnière, le livre de Laure Surville est explicitement convoqué : «La sœur de Balzac nous a raconté la joie qu'il éprouva le jour où il eut cette idée, et je la trouve aussi grande ainsi que s'il l'avait eue avant de commencer son œuvre», Contre Sainte-Beuve, éd. B. de Fallois, Paris, Gallimard, coll. « Folio», 1987 [1954], p. 214

21. M. Proust, La Prisonnière, dans RTP, t. III, p. 668.

22. G. Bachelard, La Psychanalyse du feu, Paris, Gallimard, coll. «Folio / Essais », 1985, p. 36.

23. M. Proust, La Prisonnière, op. cit., p. 664. 
l'aïeul dans une intonation, un geste du petit-fils qui ne l'a pas connu24.» La ressemblance, qui ensoleille brusquement, de façon parfaitement inattendue, le paysage bien connu de la sonate, n'est pas interprétée comme une citation ou une influence, mais comme le retour d'un thème ou d'un personnage. La rêverie, qui passe dès lors de Vinteuil à Wagner, de la partition de la sonate à celle de Tristan, identifie dans l'animation, le retour des «thèmes insistants et fugaces » qui unifient les opéras du musicien allemand, le principe dynamique qui permet à l'œuvre de s'imposer à l'auditeur comme quelque chose de «réel». La rêverie du narrateur glisse, on le voit, d'une ressemblance, qui fait pont volant entre deux œuvres, à un principe de composition, le procédé du leitmotiv, qui donne à l'œuvre de Wagner une unité qualifiée d' «organique 25 ». Cette qualité «viscérale» de l'unité de composition wagnérienne fait de l'œuvre du musicien l'analogue d'une mémoire : espace dynamique de retours et de métamorphoses démesuré par l'oubli et inquiété par la lumière trouble des réminiscences. Le personnage de Siegfried, élevé dans l'ignorance de ses origines, puis victime d'un philtre d'oubli, Brünnhilde effacée de sa conscience, s'avance sur la scène enveloppé de motifs thématiques qui éveillent dans la mémoire des spectateurs les souvenirs que l'enfant de la forêt ne fait que pressentir en lui. L'amnésie de Siegfried est le corollaire de la technique du leitmotiv. Celle-ci veut, pour s'accomplir, que la mémoire soit perçue comme la véritable scène de l'opéra, le lieu dramatique où se joue, dans cette quatrième dimension de l'espace qu'est le temps 26 , l'intelligibilité de l'œuvre-monde.

La méditation proustienne suggère une parenté d'expérience entre le surgissement de l'idée chez l'artiste créateur et, chez le lecteur ou le spectateur, ce dépaysement, cette distance interne reconfigurante 27 qui exalte, sentiment de dilatation, surprise heureuse de la compréhension, saisissement devant la brusque mise en rapport d'éléments dont on ignorait qu'ils se touchent, qu'ils communiquent ensemble. Une expression solidarise et, jusqu'à un certain point, confond l'expérience créatrice et l'expérience lectrice, la scène génétique et celle de la réception : «l'illumination rétrospective».

\section{Seconde fable : le «Conte des 365 appartements »}

Une jeune femme prend une clef dans un tiroir et fait signe à son amant de la suivre. «Venez! Je vais vous montrer quelque chose ${ }^{28}$.» Le couple s'engage dans un long couloir au bout duquel une porte, dissimulée derrière un rideau de velours, ouvre sur un débarras. La maîtresse des lieux fait jouer une serrure dans la pénombre encombrée de cartons et de malles : elle s'engage dans un nouveau couloir, traverse une antichambre, hésite sur le seuil d'un salon - derrière la porte se devinent des bruits de conversation; elle bifurque et pénètre dans une penderie. Le narrateur soupçonne, «à certains indices », qu'ils viennent de passer dans un autre appartement. Une petite porte, dissimulée entre les placards, ouvre sur un couloir assez encombré, s'élargissant, au milieu de sa longueur, en «un petit rond-point

\footnotetext{
24.Ibid., p. 664-665.

25.Ibid., p. 665

26. Proust décrit l'église de Combray comme « un espace à quatre dimensions - la quatrième étant celle du temps » (Du côté de chez Swann, dans RTP, t. I, p. 60).

27. Voir C. Pradeau, « Le décloisonnement du monde », Romantisme, n 136, juin 2007, p. 53-64.

28. J. Romains, Vorge contre Quinette, dans Les Hommes de bonne volonté, éd. O. Rony, Paris, Robert Laffont,
} coll. «Bouquins », 4 vol., 1988, t. III, p. 400-407. 
qu'éclairait une fenêtre, qui donnait sur la rue». Le narrateur découvre seulement alors qu'il a quitté l'immeuble de sa maîtresse pour un autre situé assez loin de là, dans une rue voisine. La femme interrompt les démonstrations d'étonnement de son compagnon et, désignant une vaste galerie éclairée de vitraux de couleurs, rompt le silence qu'elle a gardé tout au long de leur traversée clandestine des espaces cloisonnés de la vie privée : «Nous pourrions continuer ainsi... mais là-bas au bout il faudrait prendre un escalier... Comme cela, vous avez déjà une idée, n'est-ce pas?»

Nous sommes à Paris, au printemps 1919, dans un roman de Jules Romains, Vorge contre Quinette, dix-septième des vingt-sept tomes que comptent Les Hommes de bonne volonté, ou plus exactement, puisque le texte bénéficie de l'autonomie ambiguë des récits enchâssés, dans le «Conte des 365 appartements ». Romains, faisant fond sur l'imaginaire des romans-feuilletons, invite son lecteur à jouer avec l'idée d'un «grand serpent clandestin », un « système » d'appartements interconnectés, qui traverserait tout Paris, tout au moins celui du premier XIXe siècle, permettant aux initiés, à ceux de «la chaîne», d'aller de la Bastille à la barrière des Martyrs, «sans avoir à mettre le nez dehors ». Le conte participe du «mythe de Paris », tel que le définit Roger Caillois, ville insondable, aux mille replis, dont le romancier réaliste a pour vocation de déchiffrer les «mystères», en explorant les terra incognita de la vie privée et les marécages de l' «envers de l'histoire contemporaine». Le conte est présenté comme tel par le titre en petites capitales qui l'isole dans le corps du livre, mais aussi comme une anecdote personnelle par l'écrivain, le poète surréaliste Vorge, qui en fait le récit, entretenant le doute sur la véracité de l'histoire; il est enfin, et surtout, donné pour une idée de roman, «le plus beau livre qu'on aurait jamais écrit sur Paris 29 », comme Vorge qualifie l'ouvrage qu'il n'écrira pas mais auquel il fait mine de rêver un instant. Le conte contiendrait donc, en puissance, à l'état d'esquisse ou de projet, un roman de Paris, ou, plus justement sans doute, le roman de Paris, une œuvre qui serait l'aboutissement d'une voie royale de la fiction. Le Roman des 365 appartements, que le conte enchâssé appelle ou préfigure, serait, s'il pouvait être écrit, une œuvre englobante qui contiendrait en lui toute l'histoire d'un genre qu'il aurait vocation à couronner : roman du siècle du roman. La jeune femme qui fait office de cicerone, truchement d'une tradition orale incertaine, près de se perdre, est impuissante à raconter cette histoire autrement que sur un mode hypothétique ou approximatif : le scénario génétique qu'elle propose est confus, instable, l'origine de la «chaîne occulte» est incertaine, qu'il faut situer quelque part au tournant des XVIII et XIX ${ }^{\mathrm{e}}$ siècles, à l'âge d'or des conspirations, à l'époque de Laurence de Cinq-Cygne ou de Fouché, à moins que cela ne soit à celle de la Charbonnerie, des demi-soldes et des Amis de l'ABC, dans les années où Balzac commence à écrire La Comédie humaine et Hugo à rêver de Jean Valjean, une «chaîne», quoi qu'il en soit, qui aura su se maintenir jusqu' aux lendemains de la Grande Guerre, malgré les destructions causées par les travaux haussmanniens puis par ceux du tout-à-l'égout et du métropolitain, qui menacent tour à tour de la réduire à l'état de tronçons, de dénaturer l'idée d'un geste de totalisation - un réseau qui traverse tout Paris, constitué d'autant d'unités solidaires qu'il y a de jours dans l'année, qui embrasse tout à la fois, fût-ce symboliquement, la totalité de l'espace et du temps -, en contraignant ceux de la «chaîne» à se contenter de solidarités locales, confinées dans l'étroitesse relative d'un

29.Ibid., p. 407. 
quartier ou d'un pâté de maisons, enveloppées dans le souvenir vague, mais néanmoins actif, de la conception d'ensemble. Jules Romains invite à lire ce morceau de bravoure comme une façon pour lui de modéliser son entreprise romanesque, de la situer - comme il l'avait fait déjà, plus explicitement, mais aussi plus brutalement, sous la forme d'une vue cavalière, dans la préface de février 1932 des Hommes de bonne volonté30 - dans l'histoire d'une forme, le cycle romanesque, en laquelle il invite à voir l'un des principaux legs du XIX ${ }^{\mathrm{e}}$ siècle, et dont il lui semble qu'elle trouvera dans l'application des principes unanimistes son point de perfection. Comme Zola avant lui, Romains a la prétention de refaire Balzac en mieux, ce qui implique d'ajuster la forme héritée, le cycle romanesque, à la nouvelle donne historique, mais cet ajustement, exigé par la marche du temps, est également perçu comme une correction apportée au geste fondateur, que caractérisent la fougue, la puissance mais aussi la naïveté un peu fruste des temps héroïques. Au premier rang des défauts reconnus à Balzac, il faut compter le caractère non prémédité d'une œuvre qui se découvre une unité d'ensemble dans l'après-coup du regard rétrospectif. L'invention tardive du retour des personnages entraîne des replâtrages, des retouches, qui dissimulent mal le recyclage de matériaux qui n'ont pas été conçus ensemble mais appariés a posteriori, selon la logique bricoleuse du remploi. Les nouvelles et les romans qui composent La Comédie humaine tiennent les uns aux autres de la même solidarité fragile que les appartements du «grand serpent clandestin » évoqués par Jules Romains. La pérennité de l'ensemble est menacée en raison même du caractère rétrospectif de la conception. Les appartements de la «chaîne» comme les scènes de La Comédie humaine ont été réunis, ajointés par des couloirs de liaison, des escaliers dérobés, une porte ouverte au fond d'un placard, autant d'aménagements ingénieux mais qui donnent dangereusement prise à l'usure du temps. L'unité cyclique est une beauté ajoutée, autrement dit une pièce rapportée. Si on envisage les choses comme le fait Proust, en termes de «touche», alors, pour filer la métaphore picturale, il faudrait préciser que cette touche ou retouche n'est pas peinte dans la masse; elle n'est pas protégée par un étagement de glacis, hors des atteintes du temps, à sa juste place au cœur lumineux de l'œuvre, dont elle constituerait dès lors, comme le prétend Balzac, le foyer rayonnant. Le principe cohésif d'une «unité multiple» comme La Comédie humaine aurait donc, si du moins l'on en croit la leçon de Jules Romains, la labilité cosmétique des accessoires.

Le beau texte que Zola consacre à Balzac dans Les Romanciers naturalistes propose, en ouverture, une vision de La Comédie humaine en tour de Babel31. Zola, prenant appui sur la métaphore totalisante balzacienne de l'œuvre-cathédrale, la détourne pour représenter le devenir-ruines du cycle ou, pour le dire autrement, la fragilité paradoxale du monumental. Confrontée à son inévitable inachèvement, La Comédie humaine, à la mort de Balzac, encore en chantier, encombrée de poulies et d'échafaudages, est abandonnée au temps. Jouet de la pluie et du vent, qui s'insinuent par mille béances, l'édifice offre «des pans de muraille [qui] semblent devoir s'écrouler de vétusté et joncher le sol de leurs débris». Et pourtant, si le temps a emporté déjà les «scènes » tracées à la va-vite pour desserrer l'étreinte des usuriers, l'œuvre se dresse immense à l'horizon, couvrant de son ombre le genre romanesque.

30. J. Romains, Les Hommes de bonne volonté, op. cit., t. I, « Préface », p. 1-10.

31. É. Zola, Les Romanciers naturalistes, dans Euvres complètes, éd. H. Mitterand, Paris, Cercle du Livre Précieux, 15 vol., 1966-1970, t. XI, p. 25-26. 
On retrouve la même inquiétude envers la pérennité du monumental dans un passage du discours prononcé par Zola aux obsèques de Maupassant, aveu de lassitude du travailleur infatigable, dont la devise, nulla dies sine linea, pourrait être celle de tout écrivain engagé dans un chantier-monde, dans une œuvre dont l'étendue tend à se faire coextensive à l'existence, accès de doute en forme d'hommage rendu, plein de délicatesse, à l'un des grands maîtres de la forme brève :

Mais, le dirais-je? je suis parfois pris d'une inquiétude mélancolique devant les grosses productions de notre époque. Oui, ce sont de longues, de consciencieuses besognes, beaucoup de livres accumulés, un bel exemple d'obstination au travail. Seulement, ce sont là aussi des bagages bien lourds pour la gloire, et la mémoire des hommes n'aime pas se charger d'un pareil poids. De ces grandes œuvres cycliques il n'est jamais resté que quelques pages. Qui sait si l'immortalité n'est pas plutôt une nouvelle en trois cents lignes, la fable ou le conte que les écoliers des siècles futurs se transmettront, comme l'exemple inattaquable de la perfection classique ${ }^{32}$ ?

Zola, comme Proust et Romains le feront après lui, invite à voir dans le cycle, depuis le XIX ${ }^{\mathrm{e}}$ siècle finissant, la forme même d'une époque qui se sera reconnue dans l'ambition monumentale mais dont les œuvres les plus emblématiques n'en sont pas moins hantées par le fragmentaire, inquiétées par l'idée d'inachèvement et la mélancolie du devenir-ruines. Les «grandes œuvres cycliques » - rare occurrence du terme sous la plume de Zola, témoignage parmi d'autres de son entrée dans la langue dans les années 1890 - sont des constructions imposantes mais qui ont la fragilité de l'agrégat, unités hétérogènes où la partie conserve le souvenir de son autonomie antérieure; elle y gagne de sembler bien souvent prête à reprendre son indépendance, comme en nostalgie de ce que Zola appelle la «perfection classique» de l'œuvre serrée comme un poing, protégée de l'usure du temps par la force centripète de l'unité de composition. L'inquiétude à l'égard de la fragilité du monumental, partagée par tous les écrivains cycliques, rejoint la fascination pour le fiat lux de «l'illumination rétrospective», en ce que l'un et l'autre scénario, celui du surgissement et celui de la déprise, interrogent le paradoxe constitutif de l'unité cyclique, qui décloisonne en respectant murs et frontières, qui s'obstine à maintenir dans l'effet unitaire les fissures d'une ligne de séparation, à ménager pour chaque partie la possibilité d'une déhiscence.

L'unité cyclique est, par nature, instable et équivoque. Elle solidarise dans un ensemble englobant des œuvres qui, tout en étant données pour les parties d'un tout, les «scènes » d'une « comédie», les chapitres d'un vaste roman, conservent une forme d'autonomie, qualité qui, précisément, impose que l'on voie en elles des œuvres et non des tomes ou des volumes : on peut lire La Rabouilleuse ou Pot-Bouille sans avoir idée que ces romans sont liés à d'autres, que l'on peut retrouver ailleurs Joseph Bridau et Octave Mouret. Gérard Genette 33 a remarqué que l'autonomie de la partie varie d'une «œuvre composée» à l'autre, et même, peut-on ajouter, à l'intérieur de chaque «œuvre composée», telle partie dépendant plus qu'une autre de l'effet d'ensemble, à tel point que l'on peut considérer, à la limite, que chaque «œuvre composée» invente son propre mode d'intégration de la partie au tout. Les critères formels

32.É. Zola, « Discours pour les obsèques de Guy de Maupassant » [7 juillet 1893], ibid., t. XII, p. 686.

33. G. Genette, L'Euvre de l'art, éd. citée, t. I, p. 243-244. 
comme l'autonomie de la partie sont de peu de secours : l'ordre de lecture n'est jamais qu'une contrainte relative dont se jouent les pratiques. On peut décider de réserver le mot cycle aux ensembles romanesques susceptibles d'être abordés dans l'ignorance de l'ordre de lecture auctorial; et d'appeler romans-fleuves ou simplement romans ceux qui imposent une lecture suivie du premier au dernier tome. On peut, aussi bien, faire le choix d'une typologie souple, en distinguant entre les cycles à entrées multiples, formule privilégiée du XIX ${ }^{\mathrm{e}}$ siècle, comme La Comédie humaine et Les Rougon-Macquart, et cycles à entrée unique, formule qui a la préférence du siècle suivant, comme Jean-Christophe, À la recherche du temps perdu, Les Thibault ou Les Hommes de bonne volonté. De fait, on ne bascule pas mais on glisse d'une catégorie typologique à l'autre : l'ordre de lecture des Rougon-Macquart, chronique d'un règne impérial, est plus contraignant que celui, dyschronique, de La Comédie humaine, Jules Romains ménage dans les Hommes de bonne volonté des entrées de secours, des îlots d'autonomie, comme on en rencontre aussi dans la Recherche - Un amour de Swann-, dont l'idée est radicalement étrangère à Martin du Gard, qui ne prend certes pas modèle sur l'animation buissonnante de l'œuvre balzacienne, dont il avoue à Gide qu'elle figure au nombre de ses impossibilités, mais sur la puissante coulée de Tolstoï.

Aussi bien, si le cycle est une forme, celle-ci, instable et ondoyante, demande, pour être reçue comme telle, à être thématisée, à s'adosser à une dramaturgie. J'entends que les œuvres ainsi qualifiées ont au moins ceci en commun, par-delà la diversité de leurs modes d'intégration, de mettre en scène, en la dramatisant, la solidarité de la partie au tout. L'unité cyclique a ceci de paradoxal qu'elle procède d'un décloisonnement qui se montre éminemment soucieux de respecter murs et frontières. Il s'agit moins, de fait, de jeter bas que de percer une filière. Le cycle romanesque suppose une architecture compartimentée, un étagement articulé de scènes et de galeries, puisque, pour ses principaux représentants, écrire l'histoire du présent c'est procéder, en quelque manière, à un classement, mettre en ordre le monde pour le rendre lisible. Les protocoles diffèrent considérablement d'un romancier à l'autre, c'est une évidence, et l'on peut même penser qu'il existe sur ce point une rupture de Balzac à Proust ou de Zola à Romains. Les romanciers cycliques du $\mathrm{XX}^{\mathrm{e}}$ siècle tendraient à rompre avec une logique d'exposition ${ }^{34}$ qui serait le propre du XIX e siècle. Mais d'une solution formelle à l'autre, on retrouve les mêmes dispositifs dramaturgiques, un même souci de faire participer le lecteur de ce que Proust appelle «l'illumination rétrospective», ce que j'ai proposé d'appeler pour ma part la distance interne reconfigurante, cette surprise joyeuse, et néanmoins troublante, constitutive de la technique du retour des personnages, que l'on éprouve à se trouver dépaysé au sein même du familier, à découvrir, comme le narrateur proustien l'apprend de Gilberte, que l'on peut aller à Guermantes en prenant par Méséglise, que c'est même, peut-être, «la plus jolie façon 35 ». «L'illumination rétrospective» replie l'une sur l'autre, jusqu'à les confondre, l'expérience de l'écrivain et celle du lecteur. Le cycle serait dès lors cette forme où s'éprouve, dans toute la plénitude de l'équivoque, la parenté de l'écrire et du lire, la part de lecture qu'il y a dans l'écriture, la part de soi en désir d'écrire qu'il y a chez tout lecteur, décloisonnement des expériences vers laquelle Julien Gracq a su faire signe en conjoignant les gérondifs, en abattant la cloison légère d'un signe de ponctuation : en lisant en écrivant.

34. Ph. Hamon, Expositions. Littérature et architecture au XIXe siècle, Paris, José Corti, 1989.

35. M. Proust, Albertine disparue, dans RTP, t. IV, p. 268. 
Christophe Pradeau enseigne à l'université Paris-Sorbonne. Ses travaux portent sur les formes longues du roman (L'Idée de cycle romanesque : Balzac, Proust, Giono, thèse de doctorat, Paris VIII, 2000; Cycle et Collection, 2008); et sur la critique littéraire (Où est la littérature mondiale?, 2005 ; édition critique, avec A. Compagnon, des Réflexions sur la littérature de Thibaudet, 2007). Dernier livre publié : Proust à Illiers-Combray. L'Éclosion du monde, 2013.

christophe.pradeau@paris-sorbonne.fr

Résumés

Le mot cycle s'impose, à la fin du XIXe siècle, pour nommer les ensembles romanesques unifiés par des techniques comme le retour des personnages. L'article déplie d'abord l'héritage d'un mot emprunté à la terminologie des arts poétiques et à celui de la philologie. Il montre ensuite comment le cycle, qu'il est difficile de définir par des critères formels, trouve à le faire au moyen de «fables génétiques » où s'exposent, sur un registre métaphorique ou allégorique, la singularité et les enjeux d'une forme informe. Ce faisant, la pensée du cycle institue comme haut lieu réflexif le processus de l'invention, le plus souvent ramené à un instant d' «illumination rétrospective», selon un scénario circulant de Balzac à Proust, qui a la vertu de conjoindre l'expérience de l'écrivain créateur et celle du lecteur, dont le plaisir qu'il éprouve dans le compagnonnage des œuvres-monde peut être défini comme une distance interne reconfigurante.

At the end of the 19th century the word "cycle" became the accepted name for fiction works connected by shared techniques such as the return of characters. This article first presents the legacy of a word borrowed from the terminology of poetics and philology. It then shows how the cycle, difficult to define with formal criteria, can do so with "genetic fables" where the singularity and issues of a shapeless form are expressed in an allegorical or metaphorical register. By doing so, the cycle's thinking establishes the invention process as a reflective climax, usually reduced to a moment of "retrospective illumination", in keeping with a scenario circulating from Balzac to Proust, that has the merit of combining the writer's experience and that of the reader, whose pleasure in the companionship of world-works may be defined as a reconfiguring inner distance.

Ende des 19. Jahrhunderts bürgert sich die Bezeichnung „Zyklus“ ein für Romanfolgen mit durchgehenden Gemeinsamkeiten wie z. B. die Wiederkehr von Personen. Der Beitrag erläutert zunächst die Herkunft des aus Poetiken und aus der Philologie entlehnten Begriffs. Er zeigt im Weiteren wie der durch formale Kriterien schwer zu definierende Zyklus mittels „,genetischer Fabeln“ umrissen werden kann, die auf metaphorischer oder allegorischer Ebene die Einzigartigkeit wie auch die Probleme einer formlosen Form sichtbar machen. Hierdurch wird für die Idee des Zyklus der Prozess der Erfindung zum idealen Ort der Reflexion, ein Prozess, der gemäß einem bei Balzac und Proust gängigen Schema oft zurückgeführt wird auf den Augenblick einer ,,retrospektiven Erleuchtung“", was den Vorzug hat, die Erfahrung des schreibenden Autors mit der des Lesers zu verbinden. Das Vergnügen, welches dieser in der Gemeinschaft der „Werkwelten“ empfindet, kann als eine interne neugestaltende Distanz definiert werden.

\section{La beauté ajoutée cyclique}

La palabra ciclo se impuso, a finales del siglo XIX, para designar los conjuntos novelísticos, unificados a través de técnicas como la recurrencia de personajes. Este artículo presenta, en primer lugar, la herencia de esta palabra tomada de la terminología de las artes poéticas y la filología. Seguidamente, muestra como el ciclo, que es difícil de definir con criterios formales, puede ser definido a través de "fábulas genéticas", en las que se ponen de manifiesto -en un registro metafórico o alegórico- la singularidad y el interés de una forma informe. De este modo, el concepto de ciclo instituye como objeto reflexivo privilegiado al proceso de invención -a menudo reducido a un instante de "iluminación retrospectiva"-, según un esquema que circula desde Balzac hasta Proust, y que tiene la ventaja de conjugar la experiencia del escritor creador con la del lector, quien experimenta un placer en la camaradería de las obras-mundo que puede ser definido como una distancia interna reconfigurante.

O termo ciclo impôs-se no final do séc. XIX para nomear conjuntos romanescos unificados por técnicas tais como o retorno de personagens. Este artigo começa por traçar o legado de um termo tomado à terminologia das poéticas e da filologia. Examina em seguida como o ciclo, que é difícil de definir por critérios formais, procura fazê-lo com o recurso a "fábulas genéticas" que revelam, em registo metafórico ou alegórico, a singularidade e as dificuldades de uma forma informe. Nesta ordem, a ideia de ciclo institui como ponto alto de reflexão o processo de invenção, tantas vezes resumido a um momento de «iluminação retrospectiva», de acordo com um cenário que circula de Balzac a Proust, e em que se conjugam a experiência criativa do escritor com a do leitor, cujo prazer sentido junto a obras-mundos se pode definir como uma distância interna que é reconfigurante.

La parola ciclo si afferma alla fine del XIX secolo per indicare gli insiemi romanzeschi caratterizzati da tecniche quali il ritorno dei personaggi. L'articolo spiega innanzitutto l'eredità di un vocabolo preso in prestito dalla terminologia delle arti poetiche e dalla filologia. In seguito, mostra come il ciclo, difficile da definire secondo criteri formali, si può determinare per mezzo di "favole genetiche" che espongono, in un registro metaforico o allegorico, la particolarità e le problematiche di una forma informe. In questo modo, il pensiero del ciclo istituisce come vertice riflessivo il processo dell'invenzione, spesso ridotto ad un istante di "illuminazione retrospettiva", secondo uno scenario diffuso da Balzac a Proust, che ha la proprietà di congiungere l'esperienza dello scrittore creatore e quella del lettore, il cui piacere che egli prova in compagnia delle opere-mondo può essere definito come una distanza interna riconfigurante. 